\title{
Perspectives
}

\section{Revisiting English Entrance Examinations at Japanese Universities after a Decade}

\section{Keita Kikuchi \\ Waseda University}

Since Brown and Yamashita (1995a, 1995b) criticized the high difficulty of reading passages and the discrete-point, passive nature of the university English language entrance examinations at 10 private and 10 prestigious public universities and one nationwide examination in 1994, no studies have been conducted to monitor changes in such exams. A decade later, the present study replicates Brown and Yamashita, and seeks to identify differences in entrance examinations at the same universities in 2004. Although some changes were found, the types of items, their variety, and the skills measured did not look substantially different. Reading passages still seemed very difficult and translation tasks were still often used in 2004. The present study calls for future studies to analyze entrance examinations at different universities or to be given to students of different major fields.

Brown and Yamashita (1995a, 1995b)は1993・1994年に日本で害施された有名私立・国公 立大学それぞれ 10 校の入学試験、およびセンター試験の詳細な分析である。その結果の示 すところによれば、英文の難易度は非常に高く、内容は語彙文法に傾き、読解などの受信 的な能力に重点が置かれているのであり、著者はこれを批判している。しかしその後この ような調査はほとんど行わ饥ていない。本調査は同じ大学の入学試験およびセンター試験 の分析を行い差異を検証したものである。その結果、わずかな変化は見られたものの、項 目の型、種類、測定対象となっている技能などについてはほとんど違いがなかった。読解 問題が大半を占めており、英文の難易度は高く、また英文和訳問題の量も多かった。将来 さらに多くの入学試験問題を分析する必要があることを指摘した。

JALT Journal, Vol. 28, No. 1, May, 2006 
I

n 1995, two articles were published that analyzed 21 English language entrance examinations: 10 private, 10 public, and the national a great impact on the field of second language assessment in Japan, although some criticism was leveled that the results of these articles were not very useful for teachers who need to prepare their pupils for entrance exams (O'Sullivan, 1995). In addition, the authors' recommendations for changes to the entrance exams were criticized as being fundamentally unaware of the Japanese situation (Stapleton, 1996; Yoshida, 1996a, 1996b). Nevertheless, these articles were credited with providing "a valuable and solid first step in the process of evaluating Japanese university entrance exams" (O'Sullivan, 1995, p. 257).

As Brown and Yamashita (1995a) observed, there was, at the time of their writing, a phenomenon "known as shiken jigoku, or examination hell, which describes the months and years that Japanese young people spend preparing for entrance examinations" (p. 8). However, in the ten years since the publication of their studies, the situation surrounding the entrance examinations has changed. The number of universities grew from 552 in 1994 to 702 in 2003, while the number of upper secondary students declined in the same period (Mombukagakusho, 2004a). It is also reported that new entrants to universities or junior colleges as a percentage of 18 -year-olds nationwide surged to $49.9 \%$ in 2004 from $43.3 \%$ in 1994 (Mombukagakusho, 2004b). Furthermore, in recent newspaper articles ("Universities fear," 2004; "Birthrate benefits," 2004), it was reported that all applicants may be able to enter university within three years if they are not particular about which institution they attend. According to a recent estimate by the Japanese Ministry of Education, Culture, Sports, Science and Technology, the number of applicants for admission to colleges and universities will shrink to equal the number of available spaces by 2007 . With this change in demographics, the hold that shiken jigoku has traditionally had on university applicants will likely diminish.

How have entrance examinations at the most prestigious universities been affected by changes to Japanese society over the last ten years? In the interest of seeking the answer to this question, this study serves as a replication of Brown and Yamashita's (1995b) second study for the purpose of generating data on this subject for the first time in ten years. As in their original work, this study attempts to establish "a baseline of information so that change or lack of change in the testing practices of such universities can be monitored in future years" (Brown \& Yamashita, 1995a, p. 11). 
This study was guided by five research questions adapted from Brown and Yamashita (1995b).

1. How difficult are the various reading passages used in the 2004 university English language entrance examinations?

2. Are there differences in the level of difficulty in reading passages between private and public university examinations in 1994 and 2004 ?

3. What types of items are used on the 2004 English language entrance examinations, and how varied are they?

4. Are there differences in the types of items found in private and public university examinations in 1994 and 2004?

5. What skills were measured on the 1994 and 2004 English language entrance examinations?

\section{Method}

\section{Materials}

The CD-ROM, Xam 2004 English (JC Educational Institute, 2004), was the primary source of analysis for this study. It contains English entrance examinations from 329 universities. This CD-ROM does not include the listening section for examinations at a number of private universities. In these cases, the Zenkoku Nyushi Mondai Seikai (2004), a compilation of entrance exams from 188 universities, was consulted. The same private and public universities used in Brown and Yamashita (1995a, 1995b) were chosen for this study. Although the names of each university were clearly written in the original article, it was not clear which exams for which department were selected for analysis. In order to clarify this list, one of the authors of the original study was contacted. In Table 1 below, the list of 20 universities chosen in Brown and Yamashita (1995a, $1995 b)$ is provided, using the same system of abbreviation. Since most private universities gave different entrance exams for applicants to different disciplines, all details regarding departmental exams are included. In contrast, most public universities continue to give the same entrance exams for applicants to different disciplines, although Nagoya University and Yokohama City University are notable exceptions. In these two cases, a detailed description of each departmental exam is given. Most 
public universities set two different exam dates for the convenience of applicants. Based on the advice from one of the researchers of the original article, analysis was focused on those exams that were intended to be administered to a majority of students. For instance, most of the national universities use two different tests (zenki and koki). In this study, the zenki (earlier) test, which was used for more applicants than the koki (later) test, was used for the analysis. In addition to these 20 university exams, the 2004 Daigaku Nyuushi Sentaa exam, or Center exam, which is administered nationwide and serves as an initial screening for many schools, was included in this study.

Table 1. List of 20 universities in Brown and Yamashita (1995a, 1995b)

Private

1. Aoyama Gakuin, Dept. of English

2. Doshisha University, Dept. of English

3. Keio University, Dept. of English

4. Kansai Gaidai (Gaidai), Dept. of English (Kangai)

5. Kansai University, Dept. of English

6. Kyoto University of Foreign studies, Dept. of English

(Kyoto UFS)

7. Rikkyo University, Dept. of English

8. Sophia University, Dept. of English Language and Studies

9. Tsuda University, Dept. of English

10. Waseda University, Dept. of English, School of Education

\section{Public}

11. Hitosubashi University (Hitotsu)

12. Hokkaido University

13. Kyoto University

14. Kyushu University

15. Nagoya University, School of Letters

16. Osaka University

17. Tokyo University

18. Tokyo University of Foreign Studies (TyoUFS)

19. Tokyo Municipal University (Toritsu)

20. Yokohama City University, Dept. of International Relations

\section{Procedures}


All examinations selected were analyzed using the following procedures. First, each item was coded for item type and saved in an Excel spreadsheet program. According to Brown (1996, p. 49), a test item is "the smallest unit that produces distinctive and meaningful information on a test or rating scale." All items were coded based on item types used in Brown and Yamashita (1995a, 1995b). Second, all of the reading passages were obtained from the CD-ROM, Xam 2004 English (JC Educational Institute, 2004) in Microsoft (MS) Word 2000 format. However, for a few exams, this CD-ROM did not include MS Word files. In this case, PDF files were obtained from this CD-ROM and typed into an MS Word document file.

\section{Analyses}

All of the English reading passages on the entrance examinations were analyzed using the RightWriter program (Que Software, 1990), which lists the number of words, number of unique words, percentage of unique words (type-token ratio), syllables per word, number of sentences, and words per sentence in the passage. In addition, the readability of the passages was calculated by this program using the Flesch, Flesch-Kincaid, and Fog indexes. The number of words, syllables per word, number of sentences, and words per sentence are self-explanatory statistics. The number of unique words is the number of different words used in a passage, and type-token ratio is the percentage of unique words in the passage. The Flesch, Flesch-Kincaid and Fog readability indexes estimate the reading level of passages. The Flesch scale ranges from 0 to 100. Higher numbers in this range indicate easier to read passages. The Flesch-Kincaid and Fog readability indices are often used to establish "the grade level of students for which the reading passages should be appropriate" (Brown \& Yamashita, 1995a, p. 13) in U.S. secondary schools. Although these readability indexes have been criticized by many researchers who recommend alternative instruments for use in EFL contexts (e.g., Brown, 1998; Greenfield, 2004), the present study used these readability indexes to replicate the original study. (For more on these readability indexes, see Brown and Yamashita, 1995a, and Taylor, 2004.) 


\section{Results}

1. How difficult are the various reading passages used in the 2004 university English language entrance examinations?

Tables 2 and 3 reveal the statistical data for the reading passages of the examinations at private and public universities, respectively. Table 2 (Private) and Table 3 (Public) indicate that all universities, except Keio, used two or more than two reading passages in their entrance examinations. One private university, Sophia, and one public university, Tokyo, used as many as four passages. Looking at the average number of words in a passage, one notices that Keio and Tokyo University of Foreign Studies (Tokyo UFS) used relatively long passages. On the contrary, two public universities, Kyoto and Yokohama, used shorter passages, with fewer than 310 words per passage on average.

The number of sentences per passage is relatively straightforward to interpret. As Brown and Yamashita (1995b, p. 89) observed, average sentence length by examining words per sentence can be considered "a rough indication of the syntactic complexity of a passage." It indicates that the words/ sentence ratio on the Kansai exam was the shortest, while that of Nagoya was the longest.

The Flesch readability index yielded a range in the reading level of passages in the exams from "fairly easy" (70.98) at Kansai to "difficult" (30.12) at Nagoya. Taylor (2004) observes that the range of the Flesch readability index of standard English documents for native speakers of English should be from approximately 60 to 70 on average. Notice that the Flesch readability indexes of the passages from seven private universities, except for Kansai, Rikkyo, and Sophia, as well as eight public universities except for Hokkaido and Toritsu, average less than 60.00 . This suggests that these passages are fairly difficult to comprehend. The Fog index shows that those reading passages may be appropriate for native speakers ranging from the $9^{\text {th }}$ grade (Kansai) to $16^{\text {th }}$ grade (Nagoya). In the case of Nagoya, the difficulty of the selected reading passages seems to be at the college level or even the graduate-school level for native English speakers. Taylor (2004), however, observes that if the Fog index level is above 12, it indicates that the reading passage is too hard for most native speakers to read. Of the examinations administered at 20 universities, the average Fog index for all passages was above 12.00 at eleven. Brown and Yamashita observed, "The Fog index generally appears to agree with the Flesch-Kincaid one, but is consistently about two grades higher" (1995b, p. 89). This tendency occurred throughout most of this study. Judging by Flesch-Kincaid, many of the universities, ex- 
cept for Doshisha, Nagoya, Osaka, and Tokyo UFS, appeared to use reading passages easier than 12th grade, the final year of high school.

Table 2. Reading Passage Statistics for Private Universities, 2004

\begin{tabular}{|c|c|c|c|c|c|c|c|c|c|c|}
\hline & 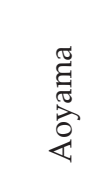 & 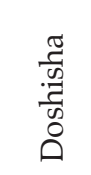 & $\stackrel{0}{\frac{0}{4}}$ & $\begin{array}{l}\tilde{\widetilde{\sigma}} \\
\infty \\
\tilde{\Xi} \\
\tilde{u}\end{array}$ & 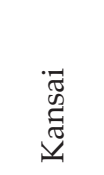 & $\begin{array}{l}n \\
\text { 岕 } \\
0 \\
0 \\
0 \\
\dot{1}\end{array}$ & 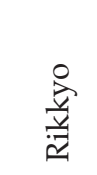 & $\begin{array}{l}\frac{\pi}{3} \\
\frac{2}{2} \\
\text { के }\end{array}$ & $\begin{array}{l}\frac{\pi}{0} \\
\frac{\omega}{2} \\
\stackrel{\omega}{n}\end{array}$ & $\begin{array}{l}\frac{\pi}{0} \\
\mathbb{D} \\
\tilde{D} \\
3 \\
3\end{array}$ \\
\hline $\begin{array}{l}\text { No. of } \\
\text { passages }\end{array}$ & 2 & 2 & 1 & 2 & 2 & 2 & 2 & 4 & 2 & 4 \\
\hline Words & 560.50 & 775.50 & 1070.003 & 373.50 & 719.00 & 539.50 & 753.00 & 601.80 & 502.00 & 399.00 \\
\hline $\begin{array}{l}\text { Unique } \\
\text { Words }\end{array}$ & 293.50 & 357.50 & 472.00 & 206.00 & 349.50 & 283.50 & 379.00 & 301.80 & 244.00 & 219.50 \\
\hline $\begin{array}{l}\text { Type-Token } \\
\text { Ratio }\end{array}$ & 0.52 & 0.46 & 0.44 & 0.55 & 0.49 & 0.53 & 0.50 & 0.50 & 0.49 & 0.55 \\
\hline $\begin{array}{l}\text { Syllables / } \\
\text { Word }\end{array}$ & 1.67 & 1.67 & 1.59 & 1.55 & 1.44 & 1.62 & 1.47 & 1.48 & 1.55 & 1.59 \\
\hline Sentences & 29.00 & 35.00 & 59.00 & 21.00 & 52.00 & 33.50 & 43.00 & 43.00 & 26.00 & 21.00 \\
\hline $\begin{array}{l}\text { Words/ } \\
\text { Sentence }\end{array}$ & 18.36 & 20.51 & 18.09 & 18.69 & 13.86 & 16.16 & 16.75 & 13.97 & 16.68 & 19.54 \\
\hline Flesch & 47.02 & 44.58 & 53.59 & 56.89 & 70.98 & 53.81 & 65.61 & 67.64 & 59.03 & 51.04 \\
\hline $\begin{array}{l}\text { Flesch } \\
\text {-Kincaid }\end{array}$ & 11.26 & 12.14 & 10.28 & 9.97 & 6.80 & 9.77 & 8.27 & 7.30 & 9.17 & 11.20 \\
\hline Fog & 13.03 & 13.85 & 12.33 & 12.48 & 9.08 & 11.74 & 9.82 & 9.24 & 11.80 & 13.38 \\
\hline
\end{tabular}

Note: All statistics for Private and Public universities as well as totals are averages.

2. Are there differences in the levels of reading passage difficulty in private and public university examinations between 1994 and 2004?

In Table 4, a statistical summary of the reading passages on the exams is presented which shows the overall mean differences between 1994 and 2004 among private and public universities as well as the Center exam. The 1994 data were obtained from Brown and Yamashita (1995b, p. 90).

In both years, public universities were found to have more reading passages, and shorter passages were used more often at public than at private universities. Furthermore, the mean for words per passage in all categories, except center exams, is higher in 2004 than in 1994, reflecting a tendency for reading passages on the exam to become longer. 
Table 3. Reading Passage Statistics for Public Universities, 2004

\begin{tabular}{|c|c|c|c|c|c|c|c|c|c|c|}
\hline & 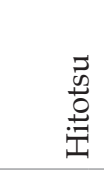 & $\begin{array}{l}\frac{0}{\pi} \\
\frac{\pi}{\pi} \\
\frac{v}{0} \\
\text { 工 }\end{array}$ & $\begin{array}{l}0 \\
0 \\
0 \\
\overrightarrow{2}\end{array}$ & $\begin{array}{l}\frac{Z}{5} \\
\frac{1}{5} \\
\frac{5}{v}\end{array}$ & 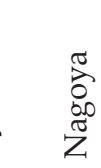 & $\begin{array}{l}\frac{\tilde{\widetilde{V}}}{\mathbb{J}} \\
\tilde{D}\end{array}$ & $\frac{\substack{\vec{y} \\
0}}{i}$ & 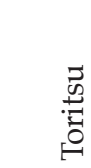 & $\begin{array}{l}\text { 号 } \\
5 \\
0 \\
\vdots \\
0 \\
0 \\
0\end{array}$ & 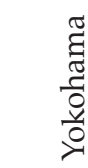 \\
\hline $\begin{array}{l}\text { No. of } \\
\text { passages }\end{array}$ & 2 & 3 & 3 & 3 & 2 & 3 & 4 & 2 & 3 & 3 \\
\hline Words & 640.00 & 409.67 & 305.67 & 454.67 & 535.50 & 314.33 & 585.00 & 545.00 & 845.00 & 265.00 \\
\hline $\begin{array}{l}\text { Unique } \\
\text { Words }\end{array}$ & 313.50 & 220.67 & 173.67 & 261.00 & 265.50 & 156.00 & 275.75 & 266.00 & 413.67 & 147.33 \\
\hline $\begin{array}{l}\text { Type-Token } \\
\text { Ratio }\end{array}$ & 0.49 & 0.54 & 0.57 & 0.57 & 0.50 & 0.50 & 0.47 & 0.49 & 0.49 & 0.56 \\
\hline $\begin{array}{l}\text { Syllables / } \\
\text { Word }\end{array}$ & 1.55 & 1.48 & 1.55 & 1.58 & 1.79 & 1.58 & 1.59 & 1.41 & 1.75 & 1.52 \\
\hline Sentences & 32.00 & 21.33 & 17.33 & 23.00 & 21.00 & 11.67 & 28.00 & 27.50 & 40.67 & 12.33 \\
\hline $\begin{array}{l}\text { Words/ } \\
\text { Sentence }\end{array}$ & 19.63 & 17.73 & 16.08 & 19.68 & 29.13 & 25.95 & 20.49 & 18.44 & 18.92 & 20.99 \\
\hline Flesch & 56.04 & 63.47 & 59.03 & 53.16 & 30.12 & 46.82 & 51.61 & 68.90 & 39.79 & 56.57 \\
\hline $\begin{array}{l}\text { Flesch } \\
\text {-Kincaid }\end{array}$ & 10.32 & 8.81 & 9.02 & 10.73 & 15.32 & 13.18 & 11.15 & 8.23 & 12.41 & 10.59 \\
\hline Fog & 11.62 & 11.21 & 11.41 & 12.88 & 16.03 & 15.75 & 13.16 & 10.82 & 14.80 & 12.42 \\
\hline
\end{tabular}

Note: All statistics for Private and Public universities as well as totals are averages.

From other statistics in this table, an overall difference between private and public universities is also apparent. The statistics on the Center exam tend to fall somewhere between these groups. However, there do not seem to be any considerable differences in the pattern between 1994 exams and 2004 exams.

3. What types of items are used on the 2004 English language entrance examinations and how varied are they?

Item types. Based on Brown and Yamashita (1995b, p. 91) and incorporating original data from this study, Tables 5 (private) and 6 (public) present a summary of different item types on the 2004 examinations. In the original study, the names of universities appear horizontally across the top of the table, while the question types, both in terms of frequency and as a percentage, appear vertically along the left side under the heading of "skill." 
Table 4. Reading Passage Statistics Summarized by University Type

\begin{tabular}{|c|c|c|c|c|c|c|c|c|}
\hline \multirow[b]{2}{*}{ Statistics } & \multicolumn{4}{|c|}{1994 Exams $^{*}$} & \multicolumn{4}{|c|}{2004 Exams } \\
\hline & Private & Public & Center & Total & Private & Public & Center & Total \\
\hline $\begin{array}{l}\text { No. of } \\
\text { universities }\end{array}$ & 10 & 10 & 1 & 21 & 10 & 10 & 1 & 21 \\
\hline $\begin{array}{l}\text { No. of } \\
\text { passages }\end{array}$ & 2.70 & 3.20 & 3.00 & 2.97 & 2.30 & 2.80 & 5.00 & 3.37 \\
\hline Words & 547.05 & 417.63 & 368.00 & 444.23 & 623.26 & 481.79 & 295.80 & 466.95 \\
\hline $\begin{array}{l}\text { Unique } \\
\text { Words }\end{array}$ & 264.82 & 222.32 & 189.67 & 225.60 & 310.63 & 249.31 & 151.20 & 237.05 \\
\hline $\begin{array}{l}\text { Type-Token } \\
\text { Ratio }\end{array}$ & 0.49 & 0.54 & 0.52 & 0.52 & 0.49 & 0.51 & 0.50 & 0.50 \\
\hline $\begin{array}{l}\text { Syllables / } \\
\text { Word }\end{array}$ & 1.51 & 1.50 & 1.49 & 1.50 & 1.46 & 1.48 & 1.40 & 1.45 \\
\hline Sentences & 28.79 & 25.18 & 24.67 & 26.21 & 33.45 & 21.98 & 19.40 & 24.94 \\
\hline $\begin{array}{l}\text { Words/ } \\
\text { Sentence }\end{array}$ & 19.51 & 17.92 & 18.77 & 18.73 & 19.04 & 22.86 & 17.00 & 19.63 \\
\hline Flesch & 59.35 & 61.63 & 61.91 & 60.96 & 63.63 & 58.20 & 71.38 & 64.40 \\
\hline $\begin{array}{l}\text { Flesch } \\
\text {-Kincaid }\end{array}$ & 9.83 & 9.11 & 9.29 & 9.41 & 9.62 & 10.98 & 8.79 & 9.79 \\
\hline Fog & 12.05 & 11.28 & 10.83 & 11.39 & 12.16 & 14.03 & 10.46 & 12.22 \\
\hline
\end{tabular}

* The 1994 data were obtained from Brown and Yamashita (1995b, p. 90)

Note: All statistics for Private and Public universities as well as totals are averages.

The method of categorization used by Brown and Yamashita (1995b, p. 91) in "reading/writing" is also used in this study. Under "translation" skills, two new categories, summarizing English sentences in Japanese $(\mathrm{E}>\mathrm{J})$ and Japanese sentences in English $(\mathrm{J}>\mathrm{E})$ have been added, since they were not question types on the 1994 examinations. Likewise, summarizing "listening" passages also appears as a new question type in this analysis.

Item variety. As Brown and Yamashita observed a decade ago, it seems that "the nature of the item types on the various university entrance examinations varies tremendously" (1995b, p. 91). This observation still applies as detailed in Tables 5 and 6. For instance, some private universities such as Kangai, Doshisha, Sophia, and Waseda place heavy emphasis on multiple-choice items, whereas some public universities such as Kyoto, Toritsu, and Tokyo UFS do not use multiple-choice items in terms of assessing reading/writing skills. In addition, while private universities such 
Table 5. Item Types on Private University Examinations, 2004

\begin{tabular}{|c|c|c|c|c|c|c|c|c|c|c|}
\hline $\begin{array}{l}\text { Skill: } \\
\text { Item type }\end{array}$ & 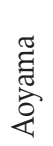 & 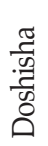 & 高 & 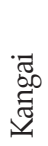 & 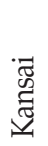 & 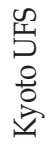 & $\frac{\grave{a}}{\frac{a}{2}}$ & $\frac{. \frac{\pi}{2}}{\frac{2}{2}}$ & $\begin{array}{l}\frac{\pi}{0} \\
\frac{0}{2} \\
\end{array}$ & $\begin{array}{l}\frac{\pi}{D} \\
\mathbb{D} \\
3 \\
3\end{array}$ \\
\hline
\end{tabular}

\section{Frequencies}

Reading/writing:

Multiple-choice

True-false

Rephrase/reorder

Fill-in

Short-answer/essay

Translation:

Translate (E->)

Translate (J->E)

Summary $(\mathrm{E}->\mathrm{J})^{*}$

Summary $(\mathrm{J}->\mathrm{E})^{*}$

Listening:

True-false

Multiple-choice

Fill-in

Dictation

Short-answer

Summary *

Total Number of Items

Percentages

Reading/writing:

Multiple-choice

True-false

Rephrase/reorder

Fill-in

$\begin{array}{rr}10 & 38 \\ 0 & 0\end{array}$

0

0

10

$\begin{array}{llllllllll}2 & 1 & 3 & 0 & 0 & 1 & 0 & 0 & 1 & 0 \\ 2 & 1 & 1 & 0 & 4 & 0 & 0 & 0 & 0 & 0 \\ 0 & 0 & 0 & 0 & 0 & 0 & 0 & 0 & 0 & 0 \\ 0 & 0 & 0 & 0 & 0 & 0 & 0 & 0 & 0 & 0\end{array}$

Short-answer/essay

Translation:

Translate (E->J)

Translate (J->E)

Summary $(\mathrm{E}->\mathrm{J})^{\star}$

Summary $(\mathrm{J}->\mathrm{E})^{*}$

Listening:

True-false

Multiple-choice

Fill-in

$\begin{array}{rr}0 & 0 \\ 10 & 0\end{array}$

$0 \quad 0$

$0 \quad 0$

$0 \quad 0$

$0 \quad 0 \quad 0$

$\begin{array}{llll}25 & 41 & 9 & 33\end{array}$

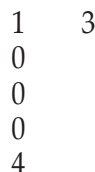

$33 \quad 35$

$\begin{array}{rrrrrr}35 & 42 & 14 & 75 & 24 & 46 \\ 0 & 0 & 0 & 0 & 0 & 0 \\ 6 & 0 & 4 & 0 & 8 & 1 \\ 0 & 3 & 9 & 0 & 0 & 0 \\ 0 & 0 & 0 & 0 & 0 & 0\end{array}$


Table 6. Item Types on Public University Examinations, 2004

\begin{tabular}{|c|c|c|c|c|c|c|c|c|c|}
\hline $\begin{array}{l}\text { Skill: } \\
\text { Item type }\end{array}$ & $\begin{array}{l}\text { ज्ञ } \\
\text { 龺 } \\
\text {. }\end{array}$ & 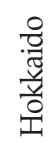 & 竞 & 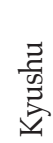 & 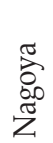 & $\begin{array}{l}\frac{\pi}{\pi} \\
\tilde{D}\end{array}$ & $\frac{\grave{\sigma}}{0}$ & 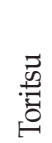 & $\begin{array}{l}\text { 号 } \\
5 \\
0 \\
\frac{\hat{\sigma}}{0}\end{array}$ \\
\hline
\end{tabular}

\section{Frequencies}

Reading/writing:

Multiple-choice

True-false

Rephrase/reorder

Fill-in

Short-answer/essay

Translation:

Translate $(\mathrm{E}->\mathrm{J})$

Translate (J->E)

Summary (E->J)*

Summary $(\mathrm{J}->\mathrm{E})^{*}$

Listening:

True-false

Multiple-choice

Fill-in

Dictation

Short-answer

Summary *

Total Number of Items

Percentages

Reading/writing:

Multiple-choice

True-false

Rephrase/reorder

Fill-in

Short-answer/essay

Translation:

Translate (E->J)

Translate (J->E)

Summary $(E->J)^{*}$

Summary $(\mathrm{J}->\mathrm{E})^{*}$

Listening:

True-false

Multiple-choice

Fill-in

Dictation

Short-answer

Summary *

Total $\%$ of Items

$\begin{array}{rrrrrrrrrr}11 & 11 & 0 & 4 & 2 & 6 & 11 & 0 & 0 & 1 \\ 0 & 0 & 0 & 0 & 0 & 0 & 0 & 0 & 10 & 0 \\ 0 & 0 & 0 & 0 & 0 & 0 & 6 & 0 & 1 & 0 \\ 5 & 4 & 0 & 0 & 3 & 0 & 3 & 4 & 1 & 9 \\ 1 & 2 & 0 & 0 & 6 & 1 & 1 & 1 & 2 & 1\end{array}$

$\begin{array}{llllllllll}5 & 5 & 7 & 11 & 6 & 6 & 5 & 10 & 4 & 1\end{array}$

$\begin{array}{llllllllll}0 & 0 & 2 & 4 & 3 & 1 & 0 & 6 & 0 & 1\end{array}$

$\begin{array}{llllllllll}1 & 0 & 0 & 0 & 0 & 0 & 1 & 0 & 1 & 1\end{array}$

$\begin{array}{llllllllll}0 & 0 & 0 & 0 & 0 & 0 & 1 & 0 & 1 & 0\end{array}$

$\begin{array}{llllllllll}0 & 0 & 0 & 0 & 0 & 0 & 0 & 0 & 0 & 0\end{array}$

$\begin{array}{llllllllll}5 & 12 & 0 & 0 & 0 & 0 & 14 & 0 & 0 & 0\end{array}$

$\begin{array}{llllllllll}0 & 0 & 0 & 0 & 0 & 0 & 6 & 0 & 0 & 0\end{array}$

$\begin{array}{llllllllll}0 & 0 & 0 & 0 & 0 & 0 & 0 & 0 & 0 & 0\end{array}$

$\begin{array}{llllllllll}0 & 0 & 0 & 0 & 0 & 0 & 0 & 0 & 6 & 0\end{array}$

$\begin{array}{llllllllll}1 & 0 & 0 & 0 & 0 & 0 & 0 & 0 & 0 & 0\end{array}$

$\begin{array}{llllllllll}29 & 34 & 9 & 19 & 20 & 14 & 48 & 21 & 26 & 14\end{array}$

$\begin{array}{llllllllll}37.9 & 32.4 & 0.0 & 21.1 & 10.0 & 42.9 & 22.9 & 0.0 & 0.0 & 7.1\end{array}$

$\begin{array}{llllllllll}0.0 & 0.0 & 0.0 & 0.0 & 0.0 & 0.0 & 0.0 & 0.0 & 38.5 & 0.0\end{array}$

$\begin{array}{llllllllll}0.0 & 0.0 & 0.0 & 0.0 & 0.0 & 0.0 & 12.5 & 0.0 & 3.8 & 0.0\end{array}$

$\begin{array}{llllllllll}17.2 & 11.8 & 0.0 & 0.0 & 15.0 & 0.0 & 6.3 & 19.0 & 3.8 & 64.3\end{array}$

$\begin{array}{llllllllll}3.4 & 5.9 & 0.0 & 0.0 & 30.0 & 7.1 & 2.1 & 4.8 & 7.7 & 7.1\end{array}$

$\begin{array}{llllllllll}17.2 & 14.7 & 77.8 & 57.9 & 30.0 & 42.9 & 10.4 & 47.6 & 15.4 & 7.1\end{array}$

$\begin{array}{llllllllll}0.0 & 0.0 & 22.2 & 21.1 & 15.0 & 7.1 & 0.0 & 28.6 & 0.0 & 7.1\end{array}$

$\begin{array}{llllllllll}3.4 & 0.0 & 0.0 & 0.0 & 0.0 & 0.0 & 2.1 & 0.0 & 3.8 & 7.1\end{array}$

$\begin{array}{llllllllll}0.0 & 0.0 & 0.0 & 0.0 & 0.0 & 0.0 & 2.1 & 0.0 & 3.8 & 0.0\end{array}$

$\begin{array}{llllllllll}0.0 & 0.0 & 0.0 & 0.0 & 0.0 & 0.0 & 0.0 & 0.0 & 0.0 & 0.0\end{array}$

$\begin{array}{llllllllll}17.2 & 35.3 & 0.0 & 0.0 & 0.0 & 0.0 & 29.2 & 0.0 & 0.0 & 0.0\end{array}$

$\begin{array}{llllllllll}0.0 & 0.0 & 0.0 & 0.0 & 0.0 & 0.0 & 12.5 & 0.0 & 0.0 & 0.0\end{array}$

$\begin{array}{llllllllll}0.0 & 0.0 & 0.0 & 0.0 & 0.0 & 0.0 & 0.0 & 0.0 & 0.0 & 0.0\end{array}$

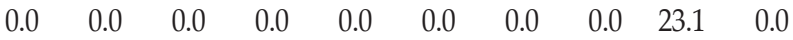

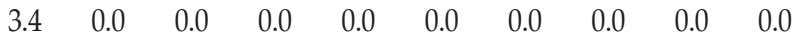

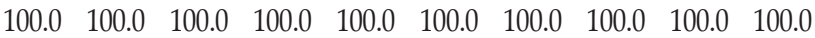

*New item types on the 2004 examinations not found on the 1994 examinations. 
as Kansai Gaidai (Kangai), Rikkyo, Sophia, and Waseda do not include any translation tasks, Kyoto, Kyushu, Osaka, and Toritsu use translation items either from English to Japanese or Japanese to English in more than $50 \%$ of their test items.

4. Are there differences in the types of items found in private and public university examinations in 1994 and 2004?

Table 7 summarizes the variety of item types on entrance examinations in 1994 and 2004 among private universities, public universities, and Center exams. Data from the 1994 exam in this table was obtained from Brown and Yamashita (1995b, p. 93).

For the most part, the same item types were used in 1994 and 2004; however, there were some interesting differences. Notably, fewer shortanswer/ essay items were used in 2004 than in 1994. This finding applies particularly to public universities, where short-answer/essay question response items accounted for just $6.82 \%$ of question types in 2004, down from $17.50 \%$ in 1994 . On the contrary, listening items using multiplechoice were used by public universities more in 2004 (8.17\%) than in 1994 $(1.25 \%)$. As observed in the original study, public and private universities tend to weigh item types differently in 2004 . For instance, while private universities used predominantly multiple-choice items $(71.69 \%)$, public universities used this item type far less $(17.43 \%)$. Translation items were used more frequently at public universities than private ones.

In addition, three new items, translating a summary from English to Japanese, translating a summary from Japanese to English, and the creation of a summary based on listening passages, appeared. None of these item types were used on the 1994 exams.

5. What skills were measured on the 1994 and 2004 English language entrance examinations?

In Tables 8 and 9, three kinds of comparisons are shown for the entrance examinations of private and public universities respectively. Based on Brown and Yamashita (1995a, 1995b), test items were categorized as a) discrete-point or integrative in nature, b) used to examine receptive or productive skills of English or translation skills, and c) dependent on passages or not. For a complete explanation of these descriptions, see Brown and Yamashita (1995a, pp. 9-11). 
Table 7. Item Type Variety Summarized by University Type

\begin{tabular}{|c|c|c|c|c|c|c|c|c|}
\hline \multirow{2}{*}{$\begin{array}{l}\text { Skill: } \\
\text { Item type }\end{array}$} & \multicolumn{4}{|c|}{1994 Exams* } & \multicolumn{4}{|c|}{2004 Exams } \\
\hline & Private & Public & Center & Total & Private & Public & Center & Total \\
\hline \multicolumn{9}{|l|}{ Frequencies } \\
\hline \multicolumn{9}{|l|}{ Reading/writing: } \\
\hline Multiple-choice & 33.7 & 7.4 & 55 & 32.03 & 31.8 & 4.6 & 45 & 26.13 \\
\hline True-false & 0.5 & 0 & 0 & 0.17 & 0 & 1 & 0 & 0.33 \\
\hline Rephrase/reorder & 0.3 & 0.1 & 4 & 1.47 & 2 & 0.7 & 5 & 2.57 \\
\hline Fill-in & 6.5 & 1.8 & 0 & 2.77 & 1.2 & 2.9 & 0 & 2.37 \\
\hline $\begin{array}{l}\text { Short-answer/ } \\
\text { essay }\end{array}$ & 1.1 & 4 & 0 & 1.70 & 0.5 & 1.5 & 0 & 0.67 \\
\hline \multicolumn{9}{|l|}{ Translation: } \\
\hline Translate (E->J) & 1.2 & 5.1 & 0 & 2.10 & 0.8 & 6 & 0 & 2.27 \\
\hline Translate $(J->E)$ & 0.9 & 2.7 & 0 & 1.20 & 0.8 & 1.7 & 0 & 0.83 \\
\hline Summary $(E->J)^{*}$ & 0 & 0 & 0 & 0.00 & 0 & 0.4 & 0 & 0.13 \\
\hline Summary $(J->E)^{*}$ & 0 & 0 & 0 & 0.00 & 0 & 0.2 & 0 & 0.07 \\
\hline \multicolumn{9}{|l|}{ Listening: } \\
\hline True-false & 0 & 0 & 0 & 0.00 & 0 & 0 & 0 & 0.00 \\
\hline Multiple-choice & 1 & 0.5 & 0 & 0.50 & 2 & 3.1 & 0 & 1.70 \\
\hline Fill-in & 0 & 1.2 & 0 & 0.40 & 0 & 0.6 & 0 & 0.20 \\
\hline Dictation & 0.1 & 0 & 0 & 0.03 & 0.1 & 0 & 0 & 0.03 \\
\hline Short-answer & 0 & 1 & 0 & 0.33 & 0 & 0.6 & 0 & 0.20 \\
\hline Summary * & 0 & 0 & 0 & 0.00 & 0 & 0.1 & 0 & 0.03 \\
\hline $\begin{array}{l}\text { Total Number } \\
\text { of Items }\end{array}$ & 45.3 & 23.8 & 59 & 42.70 & 39.2 & 23.4 & 50 & 37.53 \\
\hline \multicolumn{9}{|l|}{ Percentages } \\
\hline \multicolumn{9}{|l|}{ Reading/writing: } \\
\hline Multiple-choice & 62.31 & 26.89 & 93.22 & 60.81 & 71.69 & 17.43 & 90.00 & 57.70 \\
\hline True-false & 1.02 & 0 & 0 & 0.34 & 0.00 & 3.85 & 0.00 & 1.28 \\
\hline Rephrase/reorder & 0.67 & 0.25 & 6.78 & 2.57 & 5.62 & 1.63 & 10.00 & 5.75 \\
\hline Fill-in & 15.62 & 6.12 & 0.00 & 7.25 & 3.87 & 13.74 & 0.00 & 7.87 \\
\hline $\begin{array}{l}\text { Short-answer/ } \\
\text { essay }\end{array}$ & 6.80 & 17.50 & 0.00 & 8.10 & 4.84 & 6.82 & 0.00 & 3.89 \\
\hline \multicolumn{9}{|l|}{$\begin{array}{l}\text { essay } \\
\text { Transtation: }\end{array}$} \\
\hline Translate (E->J) & 6.06 & 28.28 & 0 & 11.45 & 4.85 & 32.10 & 0.00 & 12.32 \\
\hline Translate (J->E) & 3.31 & 13.14 & 0 & 5.48 & 3.04 & 10.11 & 0.00 & 4.39 \\
\hline Summary $(E->J)^{*}$ & 0.00 & 0.00 & 0 & 0.00 & 0.00 & 1.65 & 0.00 & 0.55 \\
\hline Summary $(J->E)^{*}$ & 0.00 & 0.00 & 0 & 0.00 & 0.00 & 0.59 & 0.00 & 0.20 \\
\hline \multicolumn{9}{|l|}{ Listening: } \\
\hline True-false & 0 & 0 & 0 & 0.00 & 0.00 & 0.00 & 0.00 & 0.00 \\
\hline Multiple-choice & 4 & 1.25 & 0 & 1.75 & 5.79 & 8.17 & 0.00 & 4.65 \\
\hline Fill-in & 0 & 3 & 0 & 1.00 & 0.00 & 1.25 & 0.00 & 0.42 \\
\hline Dictation & 0.20 & 0 & 0 & 0.07 & 0.29 & 0.00 & 0.00 & 0.10 \\
\hline Short-answer & 0 & 3.57 & 0 & 1.19 & 0.00 & 2.31 & 0.00 & 0.77 \\
\hline Summary * & 0 & 0 & 0 & 0.00 & 0.00 & 0.34 & 0.00 & 0.11 \\
\hline Total $\%$ of Items & 100.00 & 100.00 & 100.00 & 100.00 & 100.00 & 100.00 & 100.00 & 100.00 \\
\hline
\end{tabular}

Note: All statistics for Private and Public universities as well as the total are averages. 
Among the comparisons in terms of discrete-point, integrative, and translation in Table 8 for private universities, discrete-point items predominated except for Keio which put a heavy emphasis on translation $(88.9 \%)$. On the contrary, as seen in Table 9, at public universities translation occurred more frequently whereas fewer discrete-point items were used. As many as five public universities, Kyoto, Kyushu, Nagoya, Osaka, and Toritsu, used translation on $50 \%$ or more of their test items. Keio was the only private university that used translation as frequently as public universities. Eight of ten private universities used discrete-point items for more than $90 \%$ of their test items whereas only two public universities used discrete-point items $80 \%$ or more of the time.

Next, the comparison of receptive, productive, and translation items is dealt with in Tables 8 and 9. Rikkyo was the only private university which used productive items in more than $30 \%$ of its test items, while three public universities, Tokyo, Tokyo UFS, and Yokohama, used upwards of $30 \%$.

Furthermore, most of the private and public universities made frequent use of passage-dependent items. Four private universities, Kangai, Kyoto UFS, Rikkyo and Sophia, and one public university, Yokohama, were exceptions: more than $30 \%$ of their test items were found to be passage-independent.

A comparison of 1994 and 2004 item type categories for both private and public universities as well as the Center exam is presented in Table 10. Many of the same patterns that display a contrasting tendency between private and public universities found in Tables 8 and 9 were also observed in both 1994 and 2004. Although there were a few exceptions, such as a decrease in the use of integrative, productive, and passageindependent items, a similar pattern emerged for the categories of item types used in both 1994 and 2004.

\section{Discussion and Conclusion}

The level of difficulty in terms of the Flesch, Flesch-Kincaid, and Fog readability indexes in reading passages between 1994 and 2004 entrance examinations has remained essentially unchanged. Likewise, although a few new item types, such as summarizing reading passages or listening passages, have been added since 1994, the skills being measured are fundamentally the same. Most of the test items tested receptive skills or translation skills. How can these findings be interpreted? First, as observed in the original study, many of the 2004 items were based on read- 
Table 8. Categories of Item Types on Private University Entrance Examinations, 2004

\begin{tabular}{|c|c|c|c|c|c|c|c|c|c|c|}
\hline Item Category & 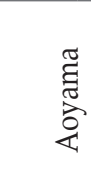 & $\begin{array}{l}\frac{\pi}{\mathcal{T}} \\
\frac{9}{7} \\
\overline{1} \\
0 \\
0\end{array}$ & $\frac{0}{d}$ & 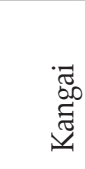 & $\begin{array}{l}\tilde{\widetilde{J}} \\
\tilde{\widetilde{T}} \\
\tilde{\Xi}\end{array}$ & 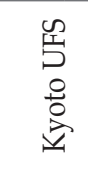 & 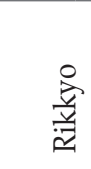 & $\begin{array}{l}\frac{\pi}{2} \\
\frac{2}{2} \\
\text { के }\end{array}$ & $\begin{array}{c}\frac{\pi}{\sigma} \\
\frac{\omega}{n} \\
\qquad\end{array}$ & $\begin{array}{l}\tilde{J} \\
\mathbb{g} \\
\tilde{J} \\
3\end{array}$ \\
\hline \multicolumn{11}{|l|}{ Frequencies } \\
\hline Discrete-point & 20 & 39 & 1 & 33 & 41 & 55 & 27 & 75 & 32 & 47 \\
\hline Integrative & 1 & 0 & 0 & 0 & 0 & 0 & 0 & 0 & 1 & 0 \\
\hline Translation & 4 & 2 & 8 & 0 & 4 & 1 & 0 & 0 & 1 & 0 \\
\hline Number of Items & 25 & 41 & 9 & 33 & 45 & 56 & 27 & 75 & 34 & 47 \\
\hline Receptive & 20 & 39 & 1 & 33 & 40 & 52 & 18 & 75 & 32 & 47 \\
\hline Productive & 1 & 0 & 0 & 0 & 1 & 3 & 9 & 0 & 1 & 0 \\
\hline Translation & 4 & 2 & 8 & 0 & 4 & 1 & 0 & 0 & 1 & 0 \\
\hline Number of Items & 25 & 41 & 9 & 33 & 45 & 56 & 27 & 75 & 34 & 47 \\
\hline $\begin{array}{l}\text { Passage- } \\
\text { dependent }\end{array}$ & 22 & 41 & 8 & 13 & 45 & 26 & 18 & 50 & 25 & 47 \\
\hline $\begin{array}{l}\text { Passage- } \\
\text { independent }\end{array}$ & 3 & 0 & 1 & 20 & 0 & 30 & 9 & 25 & 9 & 0 \\
\hline Number of Items & 25 & 41 & 9 & 33 & 45 & 56 & 27 & 75 & 34 & 47 \\
\hline \multicolumn{11}{|l|}{ Percentages } \\
\hline Discrete-point & 80.0 & 95.1 & 11.1 & 100.0 & 91.1 & 98.2 & 100.0 & 100.0 & 94.1 & 100.0 \\
\hline Integrative & 4.0 & 0.0 & 0.0 & 0.0 & 0.0 & 0.0 & 0.0 & 0.0 & 2.9 & 0.0 \\
\hline Translation & 16.0 & 4.9 & 88.9 & 0.0 & 8.9 & 1.8 & 0.0 & 0.0 & 2.9 & 0.0 \\
\hline Total $\%$ of Items & 100.0 & 100.0 & 100.0 & 100.0 & 100.0 & 100.0 & 100.0 & 100.0 & 100.0 & 100.0 \\
\hline Receptive & 80.0 & 95.1 & 11.1 & 100.0 & 88.9 & 92.9 & 66.7 & 100.0 & 94.1 & 100.0 \\
\hline Productive & 4.0 & 0.0 & 0.0 & 0.0 & 2.2 & 5.4 & 33.3 & 0.0 & 2.9 & 0.0 \\
\hline Translation & 16.0 & 4.9 & 88.9 & 0.0 & 8.9 & 1.8 & 0.0 & 0.0 & 2.9 & 0.0 \\
\hline Total $\%$ of Items & 100.0 & 100.0 & 100.0 & 100.0 & 100.0 & 100.0 & 100.0 & 100.0 & 100.0 & 100.0 \\
\hline $\begin{array}{l}\text { Passage- } \\
\text { dependent }\end{array}$ & 88.0 & 100.0 & 88.9 & 39.4 & 100.0 & 46.4 & 66.7 & 66.7 & 73.5 & 100.0 \\
\hline $\begin{array}{l}\text { Passage- } \\
\text { independent }\end{array}$ & 12.0 & 0.0 & 11.1 & 60.6 & 0.0 & 53.6 & 33.3 & 33.3 & 26.5 & 0.0 \\
\hline Total $\%$ of Items & 100.0 & 100.0 & 100.0 & 100.0 & 100.0 & 100.0 & 100.0 & 100.0 & 100.0 & 100.0 \\
\hline
\end{tabular}


Table 9. Categories of Item Types on Public University Entrance Examinations, 2004

\begin{tabular}{|c|c|c|c|c|c|c|c|c|c|c|}
\hline Item Category & 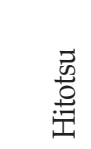 & 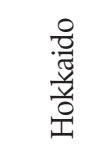 & 总 & $\begin{array}{l}z \\
z \\
\underline{5} \\
\underline{5}\end{array}$ & $\begin{array}{l}\widetilde{\sigma} \\
\underset{\widetilde{d}}{\tilde{Z}} \\
\tilde{z}\end{array}$ & $\begin{array}{l}\frac{\pi}{\pi} \\
0 \\
0\end{array}$ & $\frac{\stackrel{8}{0}}{0}$ & 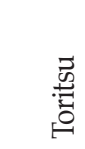 & $\begin{array}{l}0 \\
5 \\
5 \\
0 \\
0 \\
0 \\
0\end{array}$ & 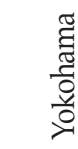 \\
\hline \multicolumn{11}{|l|}{ Frequencies } \\
\hline Discrete-point & 21 & 28 & 0 & 4 & 7 & 6 & 40 & 4 & 10 & 5 \\
\hline Integrative & 2 & 1 & 0 & 0 & 0 & 1 & 2 & 0 & 12 & 5 \\
\hline Translation & 6 & 5 & 9 & 15 & 13 & 7 & 6 & 17 & 4 & 4 \\
\hline Number of Items & 29 & 34 & 9 & 19 & 20 & 14 & 48 & 21 & 26 & 14 \\
\hline Receptive & 16 & 23 & 0 & 4 & 3 & 6 & 26 & 1 & 11 & 1 \\
\hline Productive & 7 & 6 & 0 & 0 & 4 & 1 & 16 & 3 & 11 & 9 \\
\hline Translation & 6 & 5 & 9 & 15 & 13 & 7 & 6 & 17 & 4 & 4 \\
\hline Number of Items & 29 & 34 & 9 & 19 & 20 & 14 & 48 & 21 & 26 & 14 \\
\hline $\begin{array}{l}\text { Passage- } \\
\text { dependent }\end{array}$ & 23 & 34 & 7 & 15 & 17 & 12 & 41 & 21 & 23 & 8 \\
\hline $\begin{array}{l}\text { Passage- } \\
\text { independent }\end{array}$ & 6 & 0 & 2 & 4 & 3 & 2 & 7 & 0 & 3 & 6 \\
\hline Number of Items & 29 & 34 & 9 & 19 & 20 & 14 & 48 & 21 & 26 & 14 \\
\hline \multicolumn{11}{|l|}{ Percentages } \\
\hline Discrete-point & 72.4 & 82.4 & 0.0 & 21.1 & 35.0 & 42.9 & 83.3 & 19.0 & 38.5 & 35.7 \\
\hline Integrative & 6.9 & 2.9 & 0.0 & 0.0 & 0.0 & 7.1 & 4.2 & 0.0 & 46.2 & 35.7 \\
\hline Translation & 20.7 & 14.7 & 100.0 & 78.9 & 65.0 & 50.0 & 12.5 & 81.0 & 15.4 & 28.6 \\
\hline Total $\%$ of Items & 100.0 & 100.0 & 100.0 & 100.0 & 100.0 & 100.0 & 100.0 & 100.0 & 100.0 & 100.0 \\
\hline Receptive & 55.2 & 67.6 & 0.0 & 21.1 & 15.0 & 42.9 & 54.2 & 4.8 & 42.3 & 7.1 \\
\hline Productive & 24.1 & 17.6 & 0.0 & 0.0 & 20.0 & 7.1 & 33.3 & 14.3 & 42.3 & 64.3 \\
\hline Translation & 20.7 & 14.7 & 100.0 & 78.9 & 65.0 & 50.0 & 12.5 & 81.0 & 15.4 & 28.6 \\
\hline Total $\%$ of Items & 100.0 & 100.0 & 100.0 & 100.0 & 100.0 & 100.0 & 100.0 & 100.0 & 100.0 & 100.0 \\
\hline $\begin{array}{l}\text { Passage- } \\
\text { dependent }\end{array}$ & 79.3 & 100.0 & 77.8 & 78.9 & 85.0 & 85.7 & 85.4 & 100.0 & 88.5 & 57.1 \\
\hline $\begin{array}{l}\text { Passage- } \\
\text { independent }\end{array}$ & 20.7 & 0.0 & 22.2 & 21.1 & 15.0 & 14.3 & 14.6 & 0.0 & 11.5 & 42.9 \\
\hline Total $\%$ of Items & 100.0 & 100.0 & 100.0 & 100.0 & 100.0 & 100.0 & 100.0 & 100.0 & 100.0 & 100.0 \\
\hline
\end{tabular}


Table 10. Categories of Item Types Summarized by University Type

\begin{tabular}{|c|c|c|c|c|c|c|c|c|}
\hline \multirow[b]{2}{*}{ Frequencies } & \multicolumn{4}{|c|}{1994 Exams } & \multicolumn{4}{|c|}{2004 Exams } \\
\hline & Private & Public & Center & Total & Private & Public & Center & Total \\
\hline Discrete-point & 42.00 & 11.00 & 59.00 & 37.33 & 37.00 & 12.50 & 50.00 & 33.17 \\
\hline Integrative & 1.20 & 5.00 & 0.00 & 2.07 & 0.20 & 2.30 & 0.00 & 0.83 \\
\hline Translation & 2.10 & 7.80 & 0.00 & 3.30 & 2.00 & 8.60 & 0.00 & 3.53 \\
\hline Number of Items & 45.30 & 23.80 & 59.00 & 42.70 & 39.20 & 23.40 & 50.00 & 37.53 \\
\hline Receptive & 35.50 & 8.00 & 59.00 & 34.17 & 35.70 & 9.10 & 50.00 & 31.60 \\
\hline Productive & 7.70 & 8.00 & 0.00 & 5.23 & 1.50 & 5.70 & 0.00 & 2.40 \\
\hline Translation & 2.10 & 7.80 & 0.00 & 3.30 & 2.00 & 8.60 & 0.00 & 3.53 \\
\hline Number of Items & 45.30 & 23.80 & 59.00 & 42.70 & 39.20 & 23.40 & 50.00 & 37.53 \\
\hline Passage-dependent & 25.80 & 15.20 & 14.00 & 18.33 & 29.50 & 20.10 & 21.00 & 23.53 \\
\hline $\begin{array}{l}\text { Passage- } \\
\text { independent }\end{array}$ & 19.50 & 8.60 & 45.00 & 24.37 & 9.70 & 3.30 & 29.00 & 14.00 \\
\hline Number of Items & 45.30 & 23.80 & 59.00 & 42.70 & 39.20 & 23.40 & 50.00 & 37.53 \\
\hline
\end{tabular}

Percentages

\begin{tabular}{lrrrrrrrr} 
Discrete-point & 83.63 & 37.51 & 100.00 & 73.71 & 86.97 & 43.02 & 100.00 & 76.66 \\
Integrative & 7.00 & 21.07 & 0.00 & 9.36 & 0.69 & 10.30 & 0.00 & 3.67 \\
Translation & 9.37 & 41.42 & 0.00 & 16.93 & 12.34 & 46.68 & 0.00 & 19.67 \\
Total \% of Items & 100.00 & 100.00 & 100.00 & 100.00 & 100.00 & 100.00 & 100.00 & 100.00 \\
& & & & & & & & \\
Receptive & 68.00 & 28.39 & 100.00 & 65.46 & 82.88 & 31.01 & 100.00 & 71.30 \\
Productive & 22.63 & 30.19 & 0.00 & 17.61 & 4.79 & 22.31 & 0.00 & 9.03 \\
Translation & 9.37 & 41.42 & 0.00 & 16.93 & 12.34 & 46.68 & 0.00 & 19.67 \\
Total \% of Items & 100.00 & 100.00 & 100.00 & 100.00 & 100.00 & 100.00 & 100.00 & 100.00 \\
& & & & & & & & \\
Passage-dependent & 60.48 & 68.39 & 23.73 & 50.87 & 76.96 & 83.78 & 42.00 & 67.58 \\
Passage- & & & & & & & & \\
independent & 39.52 & 31.61 & 76.27 & 49.13 & 23.04 & 16.22 & 58.00 & 32.42 \\
Total \% of Items & 100.00 & 100.00 & 100.00 & 100.00 & 100.00 & 100.00 & 100.00 & 100.00 \\
\hline
\end{tabular}

* Data of 1994 exams are obtained from Brown and Yamashita (1995b, p. 96)

Note: All statistics for Private and Public universities as well as total are averages. 
ing passages, most of which were very difficult even for native speakers of English, according to the readability indexes used in this study. As Brown and Yamashita (1995b, p. 97) state, "the ability of a given student to answer these questions will depend to some degree on high level language that is perhaps above the level of the simplified texts that are often used for pedagogical purposes in Japan." Requiring such high reading abilities for students who are just graduating from high school seems not to be ideal and probably should be avoided.

Second, given the item variety on the exams, "test-wiseness, or the ability to take tests in general, may be as important, or even more important, than the student's actual proficiency in English" (Brown \& Yamashita, 1995b, p. 97). This observation remains true for this study. For instance, in many translation tasks, I have observed that students need to translate English to Japanese using a passage of a certain length in Japanese to be successful in answering. To meet the test-makers' expectations, students probably need to know certain translation skills to construct carefully crafted pieces in Japanese. This may lead to problems of validity in that test-taking ability, rather than English proficiency, is being measured.

Third, while translation items were still abundant in the twenty 2004 examinations, only three private universities (Aoyama, Kyoto UFS, and Tsuda) and four public universities (Hitotsubashi, Hokkaido, Tokyo, and Tokyo UFS) included listening items. Since Mombukagakusho guidelines (2003) heavily promote aural/oral communication skills, more universities probably need to consider incorporating more listening items into their examinations. However, the situation is slowly changing. In 2006, the Center examination began to include listening comprehension items (National Center for University Entrance Examinations, 2004). Out of 329 the CD-ROM included, 70 examinations (approximately 21\%) included listening components.

Why haven't the entrance examinations for some prestigious universities changed very much in the last ten years? First, English departments, most of which are actually in larger literature divisions at private universities, may want to use test items such as translation more than other departments. Second, because the universities chosen for inclusion in this study are prestigious, they probably have not considered changing the format because of little perceived need to attract more applicants. They may still feel immune to the changes in the college-student demographic that universities of lower status are already facing head-on. Nevertheless, as in the case of the listening components now used at many universities, it seems that we can expect a slow pace of change in other areas of en- 
trance examinations. Ideally, the tests will also include more productive items not based on difficult reading passages.

Both the current study and Brown and Yamashita (1995a, 1995b) focused exclusively on examinations given to applicants planning to major in English at prestigious universities. It may be that major changes are underway regarding examinations for non-English majors at these and other universities. For example, it may be worthwhile to examine tests for applicants to majors related to international studies at different universities. Several areas suggest themselves for further research in the near future: (a) examining entrance examinations for different majors and/or at different universities, possibly a larger number thereof; (b) assessing vocabulary levels used in the reading passages; (c) examining the topics used; and (d) considering the valid categorization of test items, which could produce an analysis of the variety of test items along different dimensions. Readability indexes do not analyze the level of difficulty for vocabulary items or qualities such as abstractness that make reading passages complicated. Although the readability indexes used in this study did not reveal major differences, future studies using different approaches may uncover substantial differences between entrance examinations. In addition, it would be worthwhile to use a different system of categorizing items in order to investigate their complexity.

While examining numerous books published to prepare students for competitive entrance examinations, I found that cram schools ( jukus) label reading passages in terms of difficulty, for instance, "easy," "fairly easy," "difficult," or "very difficult." Furthermore, I often found that they even comment to students that the tests are getting easier or more difficult each year, although I could not find any explanation of how they analyze them. They also comment on the vocabulary levels or categorization of test items. Consulting experts in the test-preparation field would provide greater insight.

Finally, I hope that this study, as well as Brown and Yamashita's (1995a, 1995b), can become a catalyst for studies on the changes in university entrance examinations in Japan. While this study serves simply to replicate Brown and Yamashita (1995a, 1995b), I hope that future studies can develop alternative approaches to analyzing entrance examinations from different perspectives.

Keita Kikuchi is a visiting lecturer at Waseda University, School of International Liberal Studies. He holds an M.A. in ESL from University of Hawai'i at Manoa. 


\section{References}

Birthrate benefits future students. (2004, July 24). The Japan Times, p. 2.

Brown, J. D. (1996). Testing in language programs. New York: Regents/PrenticeHall.

Brown, J. D. (1998). An EFL readability index. JALT Journal, 20 (2), 7-36.

Brown, J. D., \& Yamashita, S. O. (1995a). English language tests at Japanese universities: What do we know about them? JALT Journal, 17 (1), 7-30.

Brown, J. D., \& Yamashita, S. O. (1995b). English language entrance examinations at Japanese universities: 1993 and 1994. In Brown \& Yamashita (Eds.), Language teaching in Japan (pp. 86-100). Tokyo. JALT.

Greenfield, J. (2004). Readability formulas for EFL. JALT Journal, 26 (2), 5-24.

Mombukagakusho. (2003). The course of study for foreign languages. Retrieved August 15, 2004 from http:/ / www.mext.go.jp/english/shotou/index.htm

Mombukagakusho. (2004a). School Education (Summary Tables). Retrieved August 15, 2004 from http:/ / www.mext.go.jp/english/statist/index01.htm

Mombukagakusho. (2004b).Heisei 16 nen do gakkou kihon chousa sokuhou (chousa kekka no yoshi) [The year 2004 school fundamentals study announcement (results summary)]. Retrieved August 15, 2004 from http:/ / www.mext.go.jp / b_menu/toukei/001/04073001/xls/ss0017.xls

National Center for University Entrance Examinations. (2004). Heisei 18 nendo karano daigaku nyushi sentaa shiken ni okeru eigo no Listening shiken ni tsuite [About the English listening examination on Center examination starting in 2006]. Retrieved August 15, from http://www.dnc.ac.jp/center_exam/ 17exam/elistening_test.html

O'Sullivan, B. (1995). A reaction to Brown and Yamashita "English language entrance exams at Japanese universities: What do we know about them?" JALT Journal, 17 (2), 255-257.

Que Software. (1990). Rightwriter: Intelligent grammar checker (version 4.0) [Computer software]. Sarasota, FL: Que Software.

Stapleton, P. (1996). A reaction to J. D. Brown's recent inquiry on the English entrance exam. The Language Teacher, 20 (3), 29-32.

Taylor, D. (2004). Readability info: Readability scores, grades, sentences, paragraphs, word usage, English Usage. Retrieved August 15, 2004 from http:/ / www.readability.info/

Universities fear shortage of students. (2004, July 24). The Daily Yomiuri, p. 1.

Xam 2004 English [Computer software]. Chiba: JC Educational Institute.

Yoshida, K. (1996a, January 15). Language testing in Japan: A cultural problem? The Daily Yomiuri Educational Supplement, p. 15.

Yoshida, K. (1996b, February 12). Testing the bounds of culture. The Daily Yomiuri Educational Supplement, p. 15.

Zenkoku Nyushi Mondai Seikai (2004). 2004 zenkoku nyushi mondai seikai English, private [2004 edition of university entrance exams and model answers for private universities in Japan]. Tokyo: Obunsha 